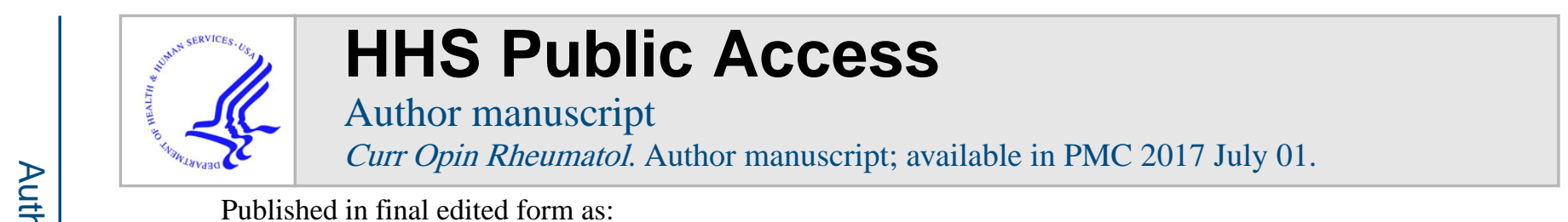

Published in final edited form as:

Curr Opin Rheumatol. 2016 July ; 28(4): 383-389. doi:10.1097/BOR.0000000000000303.

\title{
Viral Myocarditis
}

Noel R. Rose, MD, PhD

Department of Pathology, Brigham and Women's Hospital, Harvard Medical School, 77 Avenue

Louis Pasteur, NRB630OB, Boston Massachusetts 02115

\section{Abstract}

Purpose of the review-This article traces the pathways leading from viral infection of the heart by coxsackievirus B3 to autoimmune myocarditis in its various manifestations.

Recent findings-Myocarditis can be induced by a number of different infectious agents and represents a significant cause of death especially in young individuals. Following infection patients may develop lymphocytic, eosinophilic, or giant cell/granulomatous myocardial inflammation. It can lead to infectious dilated cardiomyopathy, a disease frequently requiring cardiac transplantation. Although acute viral myocarditis is frequently sub-clinical and recovery may be spontaneous, treatment of chronic myocarditis is presently unsatisfactory. Ongoing disease may be due to persistent virus in the heart or to immunopathic attack. Depending on the etiology, treatment may be antiviral or immunosuppressive. Endomyocardial biopsy is proving of value in determining etiology and deciding future therapy. A great deal of information about the pathogenesis of myocarditis has been gained from experimental models in rodents using heart disease induced by infection using coxsackievirus B3 or by immunization with cardiac myosin.

Summary-Treatment of myocarditis is still problematic and may depend on etiologic diagnosis to distinguish infectious from immune mediated disease. Both pathogenic mechanisms may co-occur in individual patients. In the future, treatment may depend upon endomyocardial biopsy, immunohistologic testing, improved imaging, and molecular genetic analysis for providing more precise diagnoses.

\section{Keywords}

Myocarditis; dilated cardiomyopathy; coxsackievirus B3; cardiac myosin

\section{Introduction}

An association between infection and autoimmune disease has long been noticed, but there are few instances where a cause and effect relationship has been firmly established in humans. Based mainly upon clinical observation and limited epidemiologic studies, viral myocarditis has long been suspected to be a primer for autoimmune heart muscle disease. My interest in this disease was occasioned by the opportunity to explore three interrelated questions. (a) Can unaltered virus cause an autoimmune disease? Previous experimental efforts to explore this question required engineering a virus to present a self-antigen or by expressing a foreign antigen in the target organ of the host. (b) What steps are responsible for the transition from an infectious process to an autoimmune disease? (c) Will experimental models be useful for improving diagnosis treatment and prevention of human 
autoimmune disease? Studies on viral myocarditis carried out in a number of laboratories have now shed considerable light on all of these questions.

\section{Viruses as a Cause of Autoimmune Myocarditis}

In an extensive historic review of the evidence, $\left(1^{* *}\right)$ points out that a number of vastly different infectious agents including viruses, bacteria, and protozoa can cause myocarditis. Among the viruses the most cited are enteroviruses, (especially coxsackievirus) adenovirus, parvovirus B19, human herpesvirus 6, human cytomegalovirus, Epstein Barr virus, and hepatitis virus. Now that aids is a treatable disease myocarditis is becoming more common in HIV infection $\left(2^{*}\right)$. As we described several years ago infection of BALB/c mice with coxsackievirus B3 (CVB3), an RNA virus, or murine cytomegalovirus (MCMV), a DNA virus, results in virtually the same inflammatory process of the heart (3). The fact that such different viruses produce the same outcome suggests that the role of infection may be nonspecific amplification ("adjuvant effect") as well as providing an antigen specific stimulus. A detailed analysis of CVB3 induced myocarditis may provide a broader picture of how viruses can produce autoimmune disease.

An up-to-date summary of our current methods of diagnosis and therapy of myocarditis is presented by Caforio et al. (4). Although formerly diagnosed mainly at autopsy, the introduction of endomyocardial biopsies has greatly expanded knowledge of the epidemiology and better refined diagnoses of myocarditis. Although exact figures of prevalence and incidence are not yet available, viral myocarditis is clearly a common disease, especially among infants, children, and young adults. Clinical diagnoses is now based mainly on cardiac function and imaging studies as well as on circulating cardiac biomarkers and antibodies. Although still relatively insensitive the endomyocardial biopsy provides additional information about histopathology immunocytochemistry and viral genome by means of PCR. (5) Increasing epidemiologic evidence suggests that a substantial number of patients with viral myocarditis progress to dilated cardiomyopathy with a substantial risk of heart failure perhaps related to effects on the autonomic nervous system $(6 *)$.

The availability of endomyocardial biopsies has brought renewed attention to the importance of persistent virus in chronic myocarditis and dilated cardiomyopathy. Many centers perform heart biopsies with a high degree of safety and with the clear benefit of guiding diagnosis and treatment although the issue is still controversial $\left(7^{*} ; 8\right)$. A review of the available evidence that persistent virus can contribute to cardiac pathology has been laid out in an extensive review of two decades of research by Fung and colleagues ( $9 * *)$. A number of in vitro mechanisms by which persistent virus can produce damage to the myocardium directly or indirectly has been described. This virus gains entry to the cell by means of the coxsackie-adenovirus receptor (CAR). And blocking of the CAR in mouse hearts completely prevents viral myocarditis. The virus encodes two proteinases, $2 \mathrm{~A}$, and $3 \mathrm{C}$ which are crucial to the completion of the full virus-like cycle, the viral proteinases contribute to intracellular and molecular damage of the myocyte. Through targeted cleavage of host proteins essential for the regulation of protein translation transcription cardiac contraction signaling transgression and a host structural proteins. CVB3 encoded proteinases $2 \mathrm{~A}$ cleaves 
dystrophin which contributes to myocardial dysfunction by increasing cell permeability and digressing forced transmission. Proteinases $2 \mathrm{~A}$ and $3 \mathrm{C}$ can also produce cytotoxicity by inducing apoptosis through direct cleavage of caspases and by inhibiting host protein translation. Oxidative stress also contributes to viral pathogenesis. Nowalany-Kozielski et al., $(10 *)$ found no significant difference between the presence of viral genomes in the biopsies studied and the immunohistochemistry results. They concluded that a high prevalence of viral genome in the myocardium of patients with dilated cardiomyopathy may not influence their long-term political outcome as manifested by death or cardiac transplantation. The final answer to the question of whether persistent virus or reactivation of latent virus is responsible for chronic myocarditis and contributes to the development of dilated cardiomyopathy requires further research.

\section{Clinical Presentation and Strategies for Treatment}

The increased use of the endomyocardial biopsy has also directed attention to the importance of differing histologic forms of myocarditis. Systematic studies of cardiac autopsies have shown that the great majority of cases of acute myocarditis are sub-clinical $\left(1^{* *}\right)$. Many others are discovered only if the patient is exposed to physical stress such as intensive athletic activity. The great majority of patients with established viral myocarditis on the basis of history, immunologic findings or other evidence is a complete recovery within a few weeks. Yet sometimes sub-clinical viral myocarditis progresses to chronic myocarditis and potentially to dilated cardiomyopathy.

In addition there is a close relationship between the endocrine system and susceptibility to myocarditis. This relationship is evident in the age predisposition of the disease occurring mainly in younger individuals, to the male to female ratio of approximately 2:1 and to the increasing recognition of peripartum myocarditis (11*).

The most common form of viral myocarditis is lymphocytic myocarditis in which the inflammatory infiltrate comprises predominantly mononuclear cells, including lymphocytes, macrophages, dendritic cells, and natural killer (NK) cells. In addition to the acute selflimiting form, lymphocytic myocarditis can become fulminant and life threatening. In addition, a proportion of lymphocytic myocarditis patients (estimated to range from $0.05 \%$ to $67 \%$ ) progresses to dilated cardiomyopathy $(1 * *)$.

Two histologically distinct forms of myocarditis are more life threatening with case fatality rates approximating 50\%. The diagnosis pathogenesis and treatment of the eosinophilic myocarditis was reviewed recently by Kuchynka et al., (12*). Although often associated with hypersensitivity responses to drugs and other allergens, many cases follow a viral infection. Vigorous treatment with corticosteroids or other immunosuppressive agents is necessary in this form of myocarditis which still represents a serious therapeutic problem. Research has been hampered by the lack of a good experimental model of the eosinophilic myocarditis until recently.

Giant cell/granulomatous myocarditis is another uncommon but frequently fatal form of acute disease that is best proved by endomyocardial biopsy (13*). This form of disease is more common in older women and individuals with another autoimmune disorder. If 
diagnosed promptly the subject seems to respond favorably to intensive immunosuppression but often requires continued treatment to avoid recurrence of the disease. There is a good model of giant cell granulomatous myocarditis in A/J mice (14).

Dilated cardiomyopathy, another possible consequence of myocarditis also requires etiologic diagnosis. Recently $\left(15^{* *}\right)$ reported on 322 patients with dilated cardiomyopathy. Based on the biopsy evidence they were divided into inflammatory/viral disease $(n=119)$ and inflammatory/viral negative $(\mathrm{n}=1053)$ groups. The numbers of patients available still precludes systematic studies of treatment but early intervention with intravenous immunoglobulins was most effective in inflammatory viral myocarditis. Early diagnosis within one year after clinical symptoms is a strong indicator of better response.

Looking forward, new approaches for therapy of heart failure in patients with inflammatory dilated cardiomyopathy is needed. Two recent reports have described progress in the use of selective immunoabsorption from patients with dilated cardiomyopathy and restoration of immunoglobulin levels with intravenous immunoglobulin. The immunoadsorption is designed to remove antibodies directed to beta-adrenergic receptors which are believed to have a pathogenic role in the disease. Reinthaler $\left(16^{* *}\right)$ compared immunoadsorption columns for both total immunoglobulin and for selective removal of the IgG3 subclass. Some patients showed surprisingly long-term benefits of this treatment. Other patients in the study who had a promising response to one such treatment could be treated repeatedly for extended benefit. Similar benefits were described by Yoshikawa et al., $\left(17^{*}\right)$ who produced symptomatic improvement in some cardiac functions even without administration of intravenous immunoglobulins. Unfortunately the specific antibody or antibodies present in the IgG3 subclass has not been defined. Interestingly Caforio et al, (18) reported that passive transfer of affinity-purified IgG from patients with dilated cardiomyopathy induced cardiac inflammation in mice.

Despite the poor reproductive capacity of the myocardium, another possible avenue to therapy under consideration for dilated cardiomyopathy is transplantation of cardiac stem cells (19). Using cardiosphere derived cells, the team at Cedars of Lebanon has continued long term studies of mini pigs receiving allogeneic cells in myocardial infarction. If proved to be successful this approach may be applicable to other cardiac diseases such as dilated cardiomyopathy.

\section{Infection VS Autoimmunity}

The present approaches to treatment of myocarditis and dilated cardiomyopathy were reviewed by Caforio et al., (20**). In addition to cardiac support the two major modalities of treatment are antiviral and immunosuppression. It should be emphasized, however, that spontaneous recovery especially from acute viral myocarditis is common and that any proposed treatment must be weighed against the benefit of standard cardiac support. A number of specific antivirals such as acyclovir and valacyclovir have been tested without definitive success but their value depends upon the selecting the specific viral agent. Remarkable improvement has been described in some patients receiving interferon-beta treatment which affects mainly enteroviral and adenoviral infections. 
With respect to immunosuppressive treatment, at least 6 controlled clinical trials have been reported since 1989. The largest of which was reported by Mason et al., (21). In a multicentered design, the investigators studied 111 patients with histopathological diagnosis of myocarditis. Based on histologic criteria and diminished left ventricular ejection fraction. The overall results did not show a significant difference in the patients who received immunosuppressive treatments compared with placebo controls. The investigators note however that some patients experienced significant benefit from immunosuppression treatment. This finding suggested that patients with an immune-mediated form of myocarditis may respond favorably to the therapy of prednisone with cyclosporine or azathioprine. Others with predominantly viral or mixed disease may show little or no benefit. Even though all patients tested were selected from a larger population of over 2233 candidates on the basis of the Dallas criteria for lymphocytic myocarditis. The population was almost certainly heterogeneous with respect to etiology.

A recent review by Frustaci and Chimenti $(22 *)$ describes the investigations of their group based on an etiologic approach to classification of patients for potential therapies. In a previous retrospective study, they compared patients who have responded favorably to immunosuppressive therapy with patients who failed to benefit from the treatment. Retrospective PCR on frozen endomyocardial biopsies showed evidence of viral genomes in the myocardium of $85 \%$ of the patients who did not respond well to immunosuppression. In contrast measurable anti-heart antibodies but no detectable virus genome was found in $90 \%$ of the favorable responders.

A more recent prospective study by these investigators expanded the results. In this study 85 patients were treated with prednisone and azathioprine and compared to 42 placebo controls. Histologic studies showed that active myocarditis with diffuse inflammatory infiltration and myocyte necrosis was present in all of the individuals in the study. Of those receiving immunosuppressive therapy, 38 of 42 showed improvement in cardiac function in contrast to none of the control patients. None of the responding patients had evidence of viral genomes in the myocardium.

These studies support the view that in the absence of continued viral presence and the production of heart specific autoantibodies, immunosuppressive therapy may be the most effective treatment of patients with lymphocytic inflammatory cardiomyopathy. Patients with evidence of persistence virus may do better with antiviral treatments. These results underline the value of identifying the etiology and tracing the pathogenesis of myocarditis as necessary clues for optimal therapy. For this purpose, models of myocarditis in rats and mice have proved to be invaluable $(23 *)$.

\section{Lessons from Experimental Models of Myocarditis}

A review by Rose and Anasyeva (24**) traced over 35 years of research designed to produce and analyze models of myocarditis. They permit mechanistic investigation of the induction and progression of disease during and following viral infection. These investigations established that mice vary in severity of viral myocarditis when infected with a cardiotropic strain of CVB3. Disease starts 2 or 3 days after infection reaches a peak approximately 9 days after inoculation and then subsides in almost all mice. Little or no evidence of cardiac 
inflammation is detectable on day 21 post infection. However, a few strains of mice especially those on a "A" or "BALB/c" genetic background develop chronic cardiac inflammation. The ongoing myocarditis in these susceptible strains was accompanied by the production of autoantibodies to cardiac antigens of which cardiac specific myosin is the most prominent. Based on the premise that CVB3 infection of susceptible mice initiates an immune process leading to autoimmune myocarditis, purified cardiac mouse myosin was administered to genetically susceptible and nonsusceptible strains of mice using purified skeletal myosin as control. The results established that autoimmune myocarditis can be induced in genetically susceptible mice by cardiac but not skeletal myosin. It can be transferred to naive recipients using CD4 T lymphocytes or, in a few mouse strains by antibody. Virus infection of the heart is necessary because strains of CVB3 that proliferate strongly in the pancreas and other organs but fail to invade the heart fail to induce myocarditis. Therefore, injury to the heart cells is needed for development of autoimmune myocarditis.

Like other experimental autoimmune diseases, myocarditis is dependent on the genetics of the host. The major histocompatibility locus of the mouse, $\mathrm{H}-2$, is an important determinant of the severity of disease induced by CVB3 infection or cardiac myosin, but multiple nonMHC immunoregulatory genes are critical in determining susceptibility (25).

An essential feature of the myosin induced model of myocarditis is that it definitively permits study of the induction, progression, and pathogenesis of autoimmune disease process in the absence of active or persistent virus (26). The analysis of the immunopathogenesis of myocarditis can be carried out on three different stages. (a.) Induction of pathogenic autoimmunity. (b.) Development of myocardial inflammation. (c.) Progression to dilated cardiomyopathy (Figure 1). Each of the three stages has a cytokine signature that mirrors both the quantity and quality of inflammation and healing (27).

Multiple factors, genetic or viral, determine whether an animal (or a human) with a coxsackie viral infection will resolve the disease completely or progress to an autoimmune sequel. As the figure indicates, at least two of the several earliest inflammatory cytokines elaborated during initial innate response to the virus are signposts of later development of an autoimmune consequence. Both cytokines IL1 beta and TNF alpha levels rise within a few hours after infection by CVB3 in the genetically susceptible strains of mice compared with non-susceptible controls. Moreover, blocking either one of these cytokines prevents the progression to autoimmune myocarditis even in genetically susceptible animals. Conversely, administration of recombinant TNF alpha or IL1 beta allows genetically "non-responsive" mice to develop the autoimmune disease.

Autoimmune myocarditis depends on a large network of interactive cytokines to determine both the severity and the composition of inflammation. It is the balance of cytokines that is the determining factor. Interferon gamma usually considered a proinflammatory cytokine actually down regulates disease so that animals deprived of this cytokine develop a particularly severe form of myocarditis. Kania et al., (28) reported that interferon gamma generated during the innate response promoted nitric oxide that limited later $\mathrm{T}$ cell proliferation. Moreover, IL17, a cytokine considered important in inflammation, regulates 
the severity of myocarditis in interferon gamma deprived animals so that they rapidly develop a fatal eosinophilic disease, providing the first mouse model for testing treatments of human eosinophilic myocarditis (29). NK cells, prominent members of the innate immune response, also limit autoimmune mediated information and fibrosis perhaps by downregulating IL17 producing T cells (30*). Most surprisingly IL17 has little impact on the severity of autoimmune myocarditis but is essential for progression to dilated cardiomyopathy (31). IL17A acts on different monocyte/macrophage populations through cardiac fibroblasts and GM-CSF production $(32 *)$.

This type of detailed analysis of cytokine profiles in autoimmune myocarditis may have two direct clinical applications. The transition from viral to autoimmune myocarditis to dilated cardiomyopathy is complex, depending on the genetics of the virus, the host, and environmental factors including the microbiome of the mouse. Yet out of the apparent chaos, the pattern of cytokines is becoming increasingly predictive of final outcome. The delineation of the cytokine profile may favor provide clues for intervention at different stages of the disease process.

\section{Conclusions}

Viral myocarditis represents a prototype of an infection that can lead to a subsequent autoimmune disease. The disease may be induced by a number of very different agents. Viral myocarditis is a relatively common, but potentially life-threatening disease, especially in young adults. Diagnoses are usually made by means of cardiac function tests and imaging studies. The rationale for endomyocardial biopsy has been strengthened by reports that treatment can be improved by the histologic, immunohistologic and molecular information derived. Treatment in the future will depend on precise diagnosis of the disease. Both persistent virus and autoimmune injury offer possible explanations for chronic myocarditis and progression to dilated cardiomyopathy. Experimental studies in murine models have shown that appropriate strains of virus in a susceptible host can trigger an autoimmune response. The progression of the response from self-limited viral myocarditis to autoimmune-mediated myocarditis and then to inflammatory dilated cardiomyopathy may in the future be predicted by assessing cytokine profiles during the disease process. Future treatment requires distinguishing infectious from autoimmune mediated disease and determining when and how to intervene in the pathogenic process.

\section{Acknowledgments}

The author's research has been supported by NIH research grants RO1 HL11308, HL067290, HL077611.

\section{References}

1**. Huber, Sally. Viral Myocarditis and Dilated Cardiomyopathy: Etiology and Pathogenesis. CPD Current Pharmaceutical Design. 22.4(2016):408-26. [With the virtual disappearance of rheumatic fever in the industrialized countries in the 1960s and 1970s, research on myocarditis waned. A few investigators, especially J. Woodruff and M. Lerner, revived interest by their studies on viral myocarditis. Sally Huber, one of the pioneers, describes the evolution of our understanding of viral myocarditis over the past 50 years.] 
2*. Lumsden, Rebecca H.; Bloomfield, Gerald S. The Causes of HIV-Associated Cardiomyopathy: A Tale of Two Worlds. BioMed Research International. 2016; (2016):1-9. [Myocarditis with essentially the same clinical presentation can be induced by a wide range of vastly different viruses including HIV. It suggests that the disease is determined mainly by the host rather than the infecting agent.]

3. Fairweather, Delisa; Kaya, Ziya; Shellam, Geoffrey R.; Lawson, Cassandra M.; Rose, Noel R. From Infection to Autoimmunity. Journal of Autoimmunity. 16.3(2001):175-86. [PubMed: 11334481]

4. Caforio ALP, Pankuweit S, Arbustini E, Basso C, Gimeno-Blanes J, Felix SB, Fu M, Helio T, Heymans S, Jahns R, Klingel K, Linhart A, Maisch B, Mckenna W, Mogensen J, Pinto YM, Ristic A, Schultheiss H-P, Seggewiss H, Tavazzi L, Thiene G, Yilmaz A, Charron P, Elliott PM. Current State of Knowledge on Aetiology, Diagnosis, Management, and Therapy of Myocarditis: A Position Statement of the European Society of Cardiology Working Group on Myocardial and Pericardial Diseases. European Heart Journal. 34.33(2013):2636-648. [PubMed: 23824828]

5. Kindermann I, Barth C, Mahfoud F, Ukena C, Lenski M, Yilmaz A, Klingel K, Kandolf R, Sechtem U, Cooper L, Bohm M. Update on Myocarditis. Journal of the American College of Cardiology. 2012:779-792. [PubMed: 22361396]

6*. Cheng, Zheng; Li-Sha, Ge; Yue-Chun, Li. Autonomic Nervous System in Viral Myocarditis: Pathophysiology and Therapy. CPD Current Pharmaceutical Design. 22.4(2016):485-98. [There is a growing body of evidence that production of autoantibodies to key components of the autonomic nervous system, such as the beta adrenergic receptor, play an important role in the progression of inflammatory myocarditis to dilated cardiomyopathy. Treatments designed to remove autoantibodies from the circulation are under investigation.]

7*. Dominguez, Fernando; Kühl, Uwe; Pieske, Burkert; Garcia-Pavia, Pablo; Tschöpe, Carsten. Update on Myocarditis and Inflammatory Cardiomyopathy: Reemergence of Endomyocardial Biopsy. Revista Española De Cardiología (English Edition). 69.2(2016):178-87. [Many investigators regard the endomyocardial biopsy as the "gold standard" for diagnosis and selection of treatment for myocarditis. This paper, and the accompanying one by Tschope and Kuhl, present the view common in Europe.]

8. Tschope C, Kuhl U. Myocarditis and inflammatory cardiomyopathy - current treatment options. Dtsch Med Wchenschr. 2016:95-102.

9**. Fung, Gabriel; Luo, Honglin; Qiu, Ye; Yang, Decheng; Mcmanus, Bruce. Myocarditis. Circulation Research. 118.3(2016):496-514. [PubMed: 26846643] [Although persistent viral genome is demonstrable in biopsies of many patients with myocarditis and infectious dilated cardiomyopathy, it is not clear whether they contribute to disease. This article reviews several decades of scholarly research by McManus and his colleagues on mechanisms by which cell injury can occur.]

10*. Nowalany-Kozielska, Ewa; Koziel, Monika; Domal-Kwiatkowska, Dorota; Wojciechowska, Celina; Jachec, Wojciech; Kawecki, Damian; Tomasik, Andrzej; Przywara-Chowaniec, Brygida; Weglarz, Ludmila; Reichman-Warmusz, Edyta; Wojnicz, Romuald. Clinical Significance of Viral Genome Persistence in the Myocardium of Patients with Dilated Cardiomyopathy. Intervirology. 2016:350-56. [This paper presents results that persisting virus in the heart may not contribute to mortality in infectious dilated cardiomyopathy.]

11*. Ciccone, Marco Matteo; Dentamaro, Ilaria; Carbonara, Santa; Ricci, Gabriella; Vestito, Domenico; Marzullo, Andrea; Tunzi, Francesco; Solarino, Biagio. Fulminant Peripartum Myocarditis Associated with Sudden Cardiac Death: A Case Report. Cardiovascular Pathology. 25.2(2016):87-89. [PubMed: 26764139] [Like most autoimmune diseases, myocarditis relates to time of hormone changes such as pregnancy. Unlike most autoimmune diseases, it is somewhat more frequent in males than females.]

12*. Kuchynka, Petr; Palecek, Tomas; Masek, Martin; Cerny, Vladimir; Lambert, Lukas; Vitkova, Ivana; Linhart, Ales. Current Diagnostic and Therapeutic Aspects of Eosinophilic Myocarditis. BioMed Research International. 2016; (2016):1-6. [Eosinophilic myocarditis is a rare but frequently fatal form of the disease. There is no well-established treatment. The first animal model of the disease was described recently, which may help in devising better treatments.]

13*. Maleszewski, Joseph J.; Orellana, Victor M.; Hodge, David O.; Kuhl, Uwe; Schultheiss, HeinzPeter; Cooper, Leslie T. Long-Term Risk of Recurrence, Morbidity and Mortality in Giant Cell 
Myocarditis. The American Journal of Cardiology. 115.12(2015):1733-738. [PubMed: 25882774] [Giant cell myocarditis, a frequently fatal form of the disease, often responds well to immunosuppressive therapy.]

14. Afanasyeva, Marina; Wang, Yan; Kaya, Ziya; Park, Sung; J. Zilliox, Michael; Schofield, Brian H.; Hill, Susan L.; Rose, Noel R. Experimental Autoimmune Myocarditis in A/J Mice Is an Interleukin-4-Dependent Disease with a Th2 Phenotype. The American Journal of Pathology. 159.1(2001):193-203. [PubMed: 11438466]

15**. Pankuweit, Sabine; Lüers, Claus; Richter, Anette; Ruppert, Volker; Gelbrich, Götz; Maisch, Bernhard. Influence of Different Aetiologies on Clinical Course and Outcome in Patients with Dilated Cardiomyopathy. Eur J Clin Invest European Journal of Clinical Investigation. 45.9(2015):906-17. [This article lays out specific criteria for earlier diagnosis and institution of treatment for patients with infectious dilated cardiomyopathy.]

16**. Reinthaler, Markus; Empen, Klaus; Herda, Lars R.; Schwabe, Anna; Rühl, Michael; Dörr, Marcus; Felix, Stefan B. The Effect of a Repeated Immunoadsorption in Patients with Dilated Cardiomyopathy after Recurrence of Severe Heart Failure Symptoms. J. Clin. Apheresis Journal of Clinical Apheresis. 30.4(2014):217-23. [PubMed: 25346275] [Several groups in Europe have been experimenting with a combination of immunoadsorption combined with intravenous immunoglobulins for treatment dilated cardiomyopathy. Some patients experience prolonged remission. Others respond favorably to repeated IA/IgG treatment.]

17*. Yoshikawa, Tsutomu; Baba, Akiyasu; Akaishi, Makoto; Wakabayashi, Yasuhisa; Monkawa, Toshiaki; Kitakaze, Masafumi; Izumi, Tohru; Tomoike, Hitonobu. Immunoadsorption Therapy for Dilated Cardiomyopathy Using Tryptophan Column-A Prospective, Multicenter, Randomized, Within-patient and Parallel-group Comparative Study to Evaluate Efficacy and Safety. J. Clin. Apheresis Journal of Clinical Apheresis. 2016 [The authors report successful use of an improved IgG3 immunoadsorbant.]

18. Caforio, Alida L.p.; Angelini, Annalisa; Blank, Miri; Shani, Alice; Kivity, Shaye; Goddard, Gisele; Doria, Andrea; Schiavo, Alessandro; Testolina, Martina; Bottaro, Stefania; Marcolongo, Renzo; Thiene, Gaetano; Iliceto, Sabino; Shoenfeld, Yehuda. Passive Transfer of Affinity-purified Antiheart Autoantibodies (AHA) from Sera of Patients with Myocarditis Induces Experimental Myocarditis in Mice. International Journal of Cardiology. 179(2015):166-77. [PubMed: 25464438]

19. Feric NT, Radisic M. Strategies and Challenges to Myocardial Replacement Therapy. Stem Cells Translational Medicine 5. 4(2016):410-16.

20**. Caforio, Alida L P.; Marcolongo, Renzo; Basso, Cristina; Iliceto, Sabino. Clinical Presentation and Diagnosis of Myocarditis. Heart. 101.16(2015):1332-344. [PubMed: 26109587] [This article lays out current protocols for diagnosing myocarditis based on immunohistologic and molecular studies of endomyocardial biopsies. The results are more sensitive than the classical Dallas criteria without apparent loss of specificity.]

21. Mason, Jay W.; O'connell, John B.; Herskowitz, Ahvie; Rose, Noel R.; Mcmanus, Bruce M.; Billingham, Margaret E.; Moon, Thomas E. A Clinical Trial of Immunosuppressive Therapy for Myocarditis. New England Journal of Medicine N Engl J Med. 333.5(1995):269-75.

22*. Frustaci, Andrea; Chimenti, Cristina. Immunosuppressive Therapy in Myocarditis. Circulation Journal Circ J. 79.1(2014):4-7. [The authors describe their experience in treating myocarditis by immunosuppression in both a retrospective and a prospective study most patients with no molecular evidence of virus infection benefited from immunosuppressive therapy.]

23*. Tajiri, Kazuko; Yasutomi, Yasuhiro; Aonuma, Kazutaka. Recent Advances in the Management of Autoimmune Myocarditis: Insights from Animal Studies. CPD Current Pharmaceutical Design. 22.4(2016):427-39. [The authors review the several experimental models of myocarditis developed over the past 30 years. Based on the pathologic mechanisms they may suggest potential therapeutic targets and approaches.]

24. Afanasyeva M, Rose N. Viral Infection and Heart Disease Autoimmune Mechanisms. Infection and Autoimmunity. 2004:299-318.

25. Li, Haiyan S.; Ligons, Davinna L.; Rose, Noel R. Genetic Complexity of Autoimmune Myocarditis. Autoimmunity Reviews. 7.3(2008):168-73. [PubMed: 18190873] 
26. Rose, Noel R. Learning from Myocarditis: Mimicry, Chaos and Black Holes. F1000Prime Rep F1000Prime Reports. 2014; 6

27. Rose, Noel R. Critical Cytokine Pathways to Cardiac Inflammation. Journal of Interferon \& Cytokine Research. 31.10(2011):705-10. [PubMed: 21861699]

28. Kania G, Siegert S, Behnke S, Prados-Rosales R, Casadevall A, Luscher TF, Luther SA, Kopf M, Eriksson U, Blyszczuk P. Innate Signaling Promotes Formation of Regulatory Nitric OxideProducing Dendritic Cells Limiting T-Cell Expansion in Experimental Autoimmune Myocarditis. Circulation. 127.23(2013):2285-294. [PubMed: 23671208]

29. Barin JG, Baldeviano GC, Talor MV, Wu L, Ong S, Fairweather D, Bedja D, Stickel NR, Fontes JA, Cardamone AB, Zheng D, Gabrielson KL, Rose NR, Cihakova D. Fatal Eosinophilic Myocarditis Develops in the Absence of IFN- and IL-17A. The Journal of Immunology 191. 8(2013):4038-047.

30*. Ong, Sufey; Ligons, Davinna L.; Barin, Jobert G.; Wu, Lei; Talor, Monica V.; Diny, Nicola; Fontes, Jillian A.; Gebremariam, Elizabeth; Kass, David A.; Rose, Noel R.; Čiháková, Daniela. Natural Killer Cells Limit Cardiac Inflammation and Fibrosis by Halting Eosinophil Infiltration. The American Journal of Pathology. 185.3(2015):847-61. [PubMed: 25622543] [This article describes continuing studies of the immunopathogenisis of eosinophilic myocarditis in the mouse.]

31. Baldeviano GC, Barin JG, Talor MV, Srinivasan S, Bedja D, Zheng D, Gabrielson K, Iwakura Y, Rose NR, Cihakova D. Interleukin-17A Is Dispensable for Myocarditis but Essential for the Progression to Dilated Cardiomyopathy. Circulation Research106. 10(2010):1646-655.

32*. Wu, Lei; Diny, Nicola L.; Ong, Sufey; Barin, Jobert G.; Hou, Xuezhou; Rose, Noel R.; Talor, Monica V.; Čiháková, Daniela. Pathogenic IL-23 Signaling Is Required to Initiate GM-CSFdriven Autoimmune Myocarditis in Mice. European Journal of Immunology Eur. J. Immunol. 46.3(2016):582-92. [PubMed: 26660726] [This article describes the steps in induction of inflammatory dilated cardiomyopathy in mice with experimental myocarditis.] 


\section{Keypoints}

- Viral infection of the myocardium is common but may be self-limiting, subclinical, or progressive and life-threatening.

- $\quad$ Present treatment is largely supportive but may require cardiac transplantation.

- $\quad$ Future treatment will likely be based on precise etiologic diagnoses and related to persistent infection, immunopathogenic signals or a mixture of the two. 
Progression from viral infection to autoimmune heart disease is cytokine-regulated

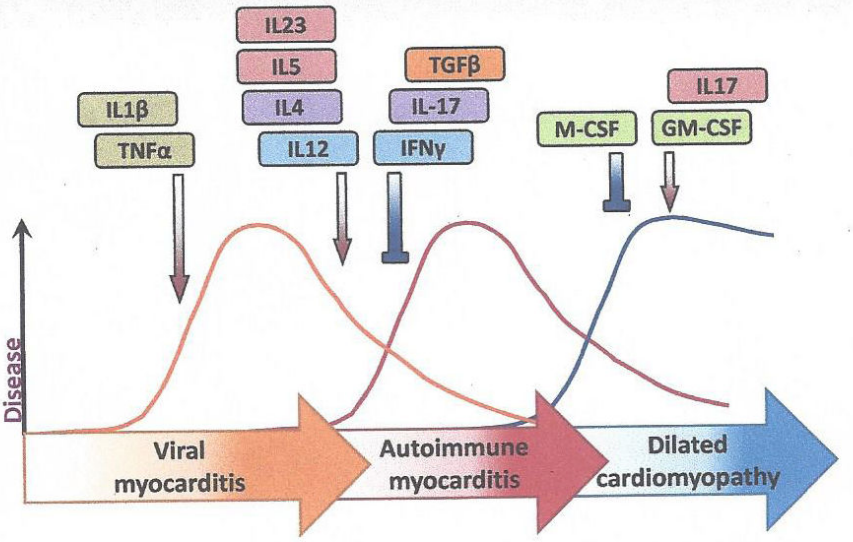

figure 1.

Dissection of steps from viral myocarditis to autoimmune myocarditis to dilated cardiomyopathy are marked by cytokine profiles. (Figure taken from Rose, 2011). 